ARTICLE

\title{
Unprecedented generation of 3D heterostructures by mechanochemical disassembly and re-ordering of incommensurate metal chalcogenides
}

\author{
Oleksandr Dolotko 1,6, Ihor Z. Hlova ${ }^{1,6}$, Arjun K. Pathak (1) 1,2,6, Yaroslav Mudryk (1) 1, Vitalij K. Pecharsky ${ }^{1,3}$, \\ Prashant Singh (10) 1,6, Duane D. Johnson (10 1,3, Brett W. Boote ${ }^{1,4}$, Jingzhe Li ${ }^{1,4}$, Emily A. Smith ${ }^{1,4}$, \\ Scott L. Carnahan ${ }^{4}$, Aaron J. Rossini ${ }^{4}$, Lin Zhou (D) ${ }^{1}$, Ely M. Eastman (i) ${ }^{1,5}$ \& Viktor P. Balema ${ }^{1 凶}$
}

Three-dimensional heterostructures are usually created either by assembling twodimensional building blocks into hierarchical architectures or using stepwise chemical processes that sequentially deposit individual monolayers. Both approaches suffer from a number of issues, including lack of suitable precursors, limited reproducibility, and poor scalability of the preparation protocols. Therefore, development of alternative methods that enable preparation of heterostructured materials is desired. We create heterostructures with incommensurate arrangements of well-defined building blocks using a synthetic approach that comprises mechanical disassembly and simultaneous reordering of layered transitionmetal dichalcogenides, $M X_{2}$, and non-layered monochalcogenides, $R E X$, where $M=T a, N b$, $\mathrm{RE}=\mathrm{Sm}$, La, and $\mathrm{X}=\mathrm{S}$, Se. We show that the discovered solid-state processes are rooted in stochastic mechanochemical transformations directed by electronic interaction between chemically and structurally dissimilar solids toward atomic-scale ordering, and offer an alternative to conventional heterostructuring. Details of composition-structure-properties relationships in the studied materials are also highlighted.

\footnotetext{
${ }^{1}$ Ames Laboratory of US Department of Energy, lowa State University, Ames, IA 50011-2416, USA. ${ }^{2}$ Department of Physics, SUNY Buffalo State, Buffalo, NY 14222, USA. ${ }^{3}$ Department of Materials Science and Engineering, lowa State University, Ames, IA 50011-1096, USA. ${ }^{4}$ Department of Chemistry, lowa State University, Ames, IA 50011-1021, USA. ${ }^{5}$ Reed College, Portland, OR 97202-8199, USA. ${ }^{6}$ These authors contributed equally: Oleksandr Dolotko, Ihor Z. Hlova, Arjun K. Pathak, Prashant Singh. ${ }^{凶}$ email: vbalema@ameslab.gov
} 
T hree-dimensional (3D) heterostructures incorporating layered van der Waals (vdW)-type metal chalcogenides into their frameworks represent a new generation of hierarchical systems with unique properties inaccessible in bulk single-phase solids ${ }^{1-4}$. Typically, 3D heterostructures are created in two fundamentally different ways. The first, known as top-down synthesis, employs two-dimensional (2D) building blocks to assemble 3Dhierarchical architectures using an appropriate additive manufacturing technique. In this case, 2D precursors are prepared by exfoliating bulk vdW materials, such as graphite, boron nitride, black phosphorus, or transition-metal dichalcogenides (TMDCs) $)^{1,2}$. The second approach, broadly defined as bottom-up synthesis, produces hierarchical 3D hetero-assemblies using stepwise chemical reactions between pure elements or small molecules that deposit individual 2D monolayers on top of each other. Often, the deposition is carried out from a gas phase $\mathrm{e}^{5-8}$ and involves transport agents ${ }^{3,4}$. While technically viable, both approaches suffer from a number of issues, including lack of suitable $2 \mathrm{D}$ precursors, insufficient process control and reproducibility, as well as poor scalability of the preparation protocols, which limit their practical applications. Hence, development of alternative methods to enable efficient preparation of $3 \mathrm{D}$ heterostructures has been identified as a major challenge for materials synthesis research ${ }^{1,9}$.

Recently, we discovered a mechanically facilitated "reshuffling" of binary TMDCs into 3D heterostructures ${ }^{10}$, where slabs of different metal dichalcogenides form layered assemblies that are tied together by weak vdW forces. The unexpected formation of hierarchical materials during mechanical milling, which is often associated with disintegration and amorphization of solids, raises an intriguing question about the ability of the uncovered phenomenon to deliver ordered 3D heterostructures, and its potential as a scalable and convenient alternative to the conventional topdown and bottom-up syntheses.

Among known 3D heterostructures, materials combining alternating layers of $\mathrm{vdW}$ TMDCs $\left(\mathrm{MX}_{2}, \mathrm{M}=\right.$ transition metal; $\mathrm{X}$ $=\mathrm{S}$ or $\mathrm{Se}$ ) and slabs of ionic rare-earth monochalcogenides (REX, $\mathrm{RE}=$ rare-earth metal) in the same superlattice are of particular interest. Such materials are often described as (REX) $)_{1+x}\left(\mathrm{MX}_{2}\right)$, where $1+x=\left(4 a_{2} / 2 a_{1}\right)$ and $a$ parameters represent the lattice parameters of the individual REX and $\mathrm{MX}_{2}$ compounds ${ }^{11}$. Periodicities of the $\mathrm{MX}_{2}$ and REX building blocks in (REX) ${ }_{1+x}\left(\mathrm{MX}_{2}\right)$ are incommensurate along the $a$-axis, but they are commensurate along two other axes, $b$ and $c$ (Fig. 1). For example, the material described by the formal chemical composition of $(\mathrm{SmS})_{1.19}\left(\mathrm{TaS}_{2}\right)$ is built from NaCl-type SmS layers alternating with $\mathrm{TaS}_{2}$ fragments in which tantalum atoms are surrounded by slightly distorted trigonal prisms of sulfur. Both structural units can be described in the $F m 2 m$ space group with lattice parameters $a_{1}=$ 5.552(3) $\AA, b_{1}=5.679(4) \AA, c_{1}=22.50(4) \AA$ for the SmS fragment, and $a_{2}=3.293(1) \AA, b_{2}=5.679(4) \AA, c_{2}=22.50(4) \AA$ for $\mathrm{TaS}_{2}$ sub-lattice. The corresponding sub-lattice axes are parallel to each other, and the layers are stacked in the $c$-direction. The $(\mathrm{SmS})_{1.19} \mathrm{TaS}_{2}$ composition of the material is governed by the ratio $a_{2} / a_{1}$ defining the number of formula units per cell ${ }^{3,12}$.

Due to this structural arrangement, $(\mathrm{REX})_{1+x}\left(\mathrm{MX}_{2}\right)$ are frequently denoted as misfit materials ${ }^{3,4}$, and the presence of chemically and structurally dissimilar 2D fragments in their crystal lattices is responsible for the emergence of intriguing physical properties, including superconductivity ${ }^{13}$, metallicity, semiconductivity $3,14,15$, and diverse magnetism $3,16,17$, among others ${ }^{4,12,18}$. The majority of $(\mathrm{REX})_{1+x}\left(\mathrm{MX}_{2}\right)$ known to date have been synthesized from pure elements, metals, and chalcogens, by heating them in sealed ampules in the presence of halogens or metal halides as transport agents,3,19-21. As exact compositions of such complex multi-component materials are hard to control during high-temperature synthesis, the conventionally prepared heterostructures are routinely denoted by the theoretical (limiting) compositions of the respective misfit compounds. In a few instances, when $(\mathrm{REX})_{1+x}\left(\mathrm{MX}_{2}\right)$ materials were investigated by quantitative analytical techniques, asobtained RE:M:X ratios deviated from the expected (theoretical misfit) values, indicating compositional irregularities in both REX and $\mathrm{MX}_{2}$ sub-lattices. In addition, the studied compounds contained noticeable amounts of halogens that served as transport agents during their synthesis, complicating the analytical results $3,4,11,20,22$.

Below, we describe an example of the mechanochemical solidstate generation of $(\mathrm{REX})_{1+x}\left(\mathrm{MX}_{2}\right)$ heterostructures under ambient conditions, whereby $(\mathrm{REX})_{1+x}\left(\mathrm{MX}_{2}\right)$ form as spatially disorganized nano-architectures. Their crystallinity substantially improves upon subsequent annealing that, however, does not alter their internal atomic arrangements, resembling in a way the behavior of mechanochemically generated molecular solids ${ }^{23}$ or coordination polymers ${ }^{24}$. Density-functional theory (DFT)
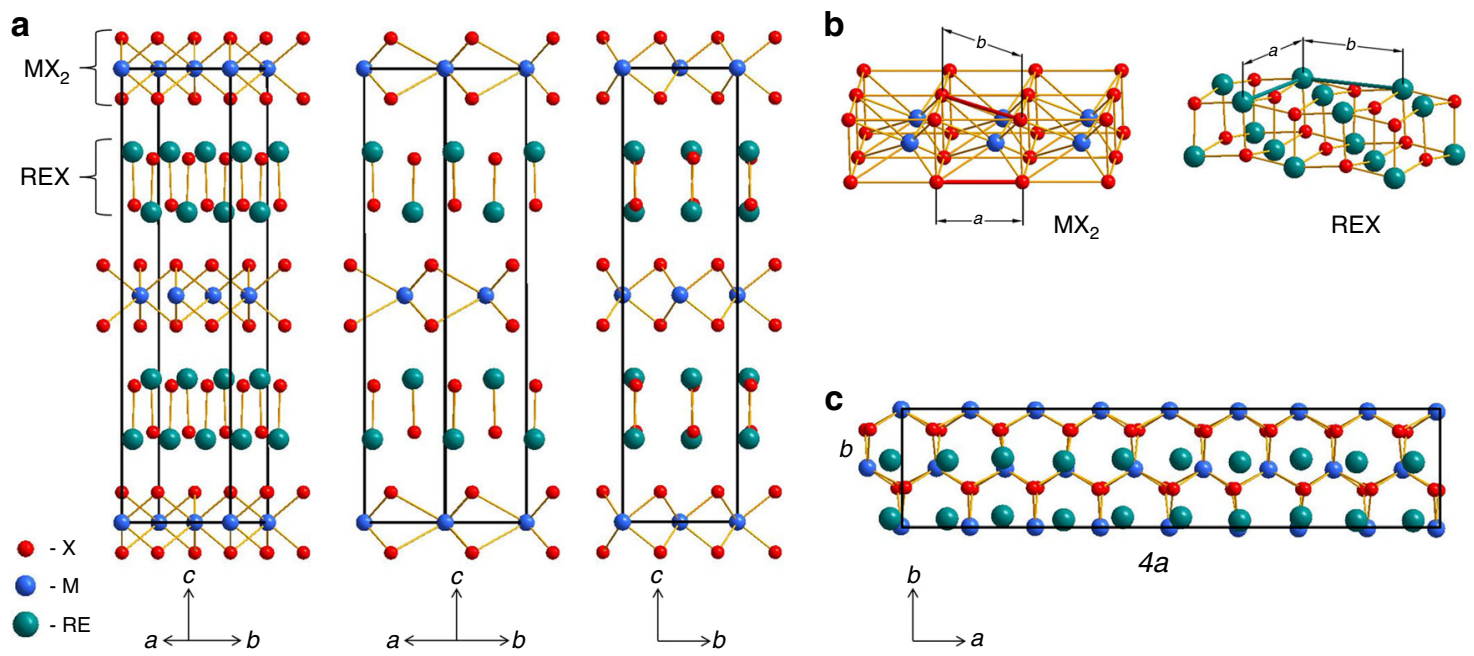

Fig. 1 Schematic illustration of 3D heterostructures. Schematic of $(R E X)_{1+x}\left(M X_{2}\right)$, where $M=$ transition metal, for example, $N b$ or Ta, $R E=$ rare-earth metal, for example, La or Sm, and X $=\mathrm{S}$ or Se. a The three different projections shown can be observed by STEM imaging. $\mathbf{b}$ Perspective views of MX $\mathrm{M}_{2}$ and REX building blocks. c Projection of the heterostructure shown in a along [0 01 1] direction highlighting incommensurability along the $a$-axis with chalcogen atoms in the REX fragments omitted for clarity. 

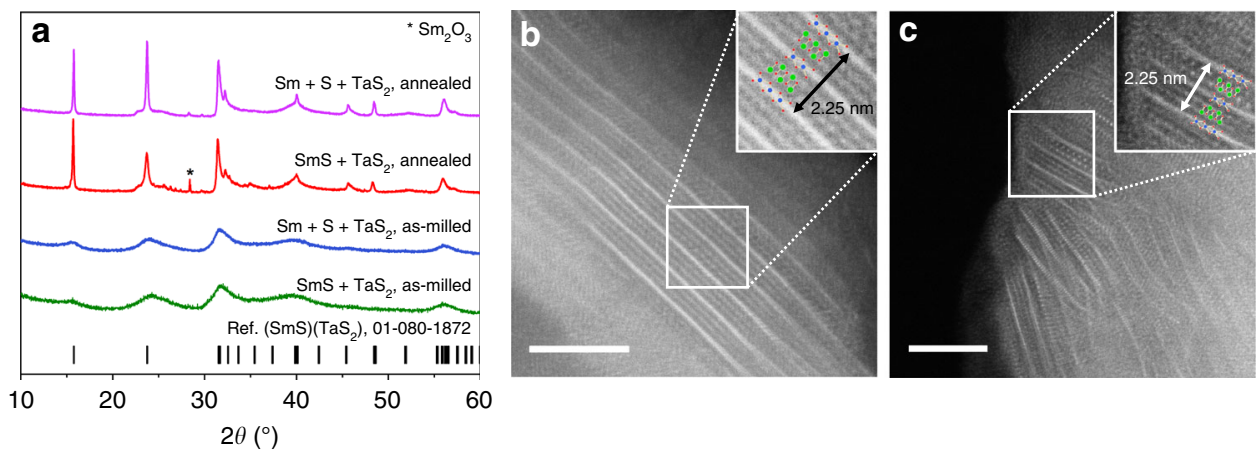

Fig. 2 Mechanochemical synthesis of the (SmS)( $\left.\mathrm{TaS}_{\mathbf{2}}\right)$ heterostructure. a PXRD patterns of $(\mathrm{SmS})\left(\mathrm{TaS}_{2}\right)$ obtained by ball milling of $\mathrm{SmS}$ and TaS ${ }_{2}$, or $\mathrm{Sm}$, $\mathrm{S}$, and $\mathrm{TaS}_{2}$ in the planetary mill for $30 \mathrm{~h}$, and of the respective materials annealed at $1000{ }^{\circ} \mathrm{C}$ for 3 days. PXRD patterns of the starting materials, SmS and $\mathrm{TaS}_{2}$, are presented in Supplementary Information. b, c HAADF-STEM images of respective as-milled samples. The insets display enhanced views of the 3D heteroatomic moieties. Scale bars, $5 \mathrm{~nm}$

calculations provide valuable insight into the discovered synthetic phenomenon and reveal details about interatomic forces that guide otherwise stochastic mechanochemical processes toward self-organization. To avoid confusion while describing our materials synthesis, the 3D heterostructures discussed are denoted as $(\mathrm{REX})_{n}\left(\mathrm{MX}_{2}\right)$, where $n$ reflects the molar amount of the REX compound, which is combined with one molar equivalent of $\mathrm{MX}_{2}$ during synthesis. For the theoretical misfit compositions, $n=1+$ $x$, where $x$ is calculated as noted above $\mathrm{e}^{11}$.

\section{Results}

Mechanochemical synthesis. Processing of an equimolar mixture of $\mathrm{SmS}$ and $\mathrm{TaS}_{2}$ in a planetary mill for $30 \mathrm{~h}$ produces a powder whose powder X-ray diffraction (PXRD) pattern (Fig. 2a) does not contain Bragg peaks of the starting materials indicating their complete conversion into the reaction products (Supplementary Figs. 1 and 2a). Instead, it consists of a set of broad Bragg reflections, positions of which agree with those expected for a $(\mathrm{SmS})_{n}\left(\mathrm{TaS}_{2}\right)$-type heterostructure. High-temperature annealing improves the crystallinity of the as-milled sample and significantly reduces the width of the Bragg reflections in its PXRD pattern without shifting their positions. The prepared $(\mathrm{SmS})\left(\mathrm{TaS}_{2}\right)$ is isostructural to $(\mathrm{PbSe})_{1.13} \mathrm{TaS}_{2}{ }^{25}$ and its REX and $\mathrm{MX}_{2}$ building blocks crystallize in the $\mathrm{Fm} 2 \mathrm{~m}$ space group. The relationship between the unit-cell parameters in $(\mathrm{SmS})\left(\mathrm{TaS}_{2}\right)$ is defined by the space fitting of the structurally dissimilar SmS and $\mathrm{TaS}_{2}$ fragments, leading to incommensurate modulation distorting periodicity along the $a$ crystallographic direction (Fig. 1). The structural parameters determined for as-milled and annealed $(\mathrm{SmS})\left(\mathrm{TaS}_{2}\right)$, as well as the modulation factors $\alpha=a[\mathrm{REX}] / a$ $\left[\mathrm{MX}_{2}\right]$, are listed in Table 1. Remarkably, they are in good agreement with the values reported in the literature as $(\mathrm{SmS})_{1.19}\left(\mathrm{TaS}_{2}\right)^{19,26}$.

$(\mathrm{SmS})\left(\mathrm{TaS}_{2}\right)$ also forms when $\mathrm{TaS}_{2}$ is combined with the equimolar amounts of pure $\mathrm{Sm}$ and $\mathrm{S}$ (1:1:1 molar ratio) and processed according to the identical preparation protocol. Both as-milled and annealed materials are practically indistinguishable from those prepared from binary compounds (Fig. 2a), suggesting a similar reaction pathway in both cases. Given the fact that SmS easily forms upon ball milling of Sm and S (Supplementary Fig. 2a), the formation of a SmS intermediate during mechanical processing of $\mathrm{Sm}, \mathrm{S}$, and $\mathrm{TaS}_{2}$ is a highly feasible first step. Also notable is the fact that although $(\mathrm{SmS})\left(\mathrm{TaS}_{2}\right)$ has been prepared with a significant $(\sim 19 \mathrm{~mol} . \%)$ excess of $\mathrm{TaS}_{2}$, no residual precursors could be detected in this material before and after the annealing, which indicates their complete conversion into the reaction products.
A sample with theoretical misfit composition $(\mathrm{SmS})_{1.19}\left(\mathrm{TaS}_{2}\right)$ 19,26 was also synthesized by ball milling $\mathrm{SmS}$ and $\mathrm{TaS}_{2}$ combined in a 1.19:1 molar ratio. While as-milled and annealed solids are similar to $(\mathrm{SmS})\left(\mathrm{TaS}_{2}\right)$, still unreacted exfoliated $\mathrm{TaS}_{2}$ nanosheets are present in the as-milled sample (Supplementary Fig. 3b), which is in line with the reaction mechanism depicted in Fig. 3. Further, the annealed $(\mathrm{SmS})_{1.19}\left(\mathrm{TaS}_{2}\right)$ contains a distinct secondary phase in its PXRD pattern. This minor, most likely samarium-oxysulfide impurity, is not present in the starting materials or in the $(\mathrm{SmS})\left(\mathrm{TaS}_{2}\right)$ product.

The high-angle annular dark-field scanning transmission electron microscopy (HAADF-STEM) and energy-dispersive Xray spectroscopy (EDS) experiments shed light on this mystery. As evident from Fig. 2b, c (and Supplementary Fig. 3b, c) asmilled $(\mathrm{SmS})\left(\mathrm{TaS}_{2}\right)$ and $(\mathrm{SmS})_{1.19}\left(\mathrm{TaS}_{2}\right)$ heterostructures are composed from similar moieties. Their spatial alignments substantially improve upon annealing, which is in line with PXRD data. The interlayer $\mathrm{TaS}_{2}-\mathrm{TaS}_{2}$ distances within the separate heterostructured moieties remain unaffected by heating, hence suggesting similarity of the atomic arrangements in asmilled and annealed materials.

Detailed HAADF-STEM and EDS investigations (Fig. 4) of a favorably oriented particle of the annealed $(\mathrm{SmS})\left(\mathrm{TaS}_{2}\right)$ reveal the presence of a $\mathrm{TaS}_{2}-\mathrm{TaS}_{2}$ double layer, suggesting that the excess of $\mathrm{TaS}_{2}$ used for its preparation is incorporated into the final product as adjacent $\mathrm{TaS}_{2}-\mathrm{TaS}_{2}$ layers, representing stacking faults. Similar structural arrangements have been observed in other particles of the mechanochemically prepared $(\mathrm{SmS})\left(\mathrm{TaS}_{2}\right)$ (Supplementary Fig. 4), and also reported in the literature for $(\mathrm{BiS})\left(\mathrm{VS}_{2}\right)_{n}$ misfit materials $(1 \leq n \leq 15)^{27}$. Besides stacking faults, vacancies, antisites, and other defects may also contribute to compositional irregularities of misfit compounds.

Thus, our results unambiguously confirm the formation of 3D misfit heterostructures upon mechanochemical disassembly and re-ordering of binary metal chalcogenide precursors. The suitable nano-sized building blocks can be also generated in situ from the elements; hence, it is expected that mechanical processing of Ta, $\mathrm{Sm}$, and $\mathrm{S}$ would produce $(\mathrm{SmS})_{n}\left(\mathrm{TaS}_{2}\right)$ hetero-assemblies as well.

To test this hypothesis, we ball milled a mixture of $\mathrm{Sm}, \mathrm{Ta}$, and $S$, combined in the 1:1:3 molar atomic ratio, in a shaker mill for $12 \mathrm{~h}$. Then, the as-milled powder was studied using HAADFSTEM and EDS elemental mapping (Supplementary Fig. 5), which confirms the presence of 3D heterostructures in the sample. However, $\mathrm{Ta}$ is incompletely dispersed in the as-milled material and Ta-rich spots remain visible in its EDS mapping (Supplementary Fig. 5c). The high-temperature annealing produces $(\mathrm{SmS})\left(\mathrm{TaS}_{2}\right)$ identical to that made from other precursors. 


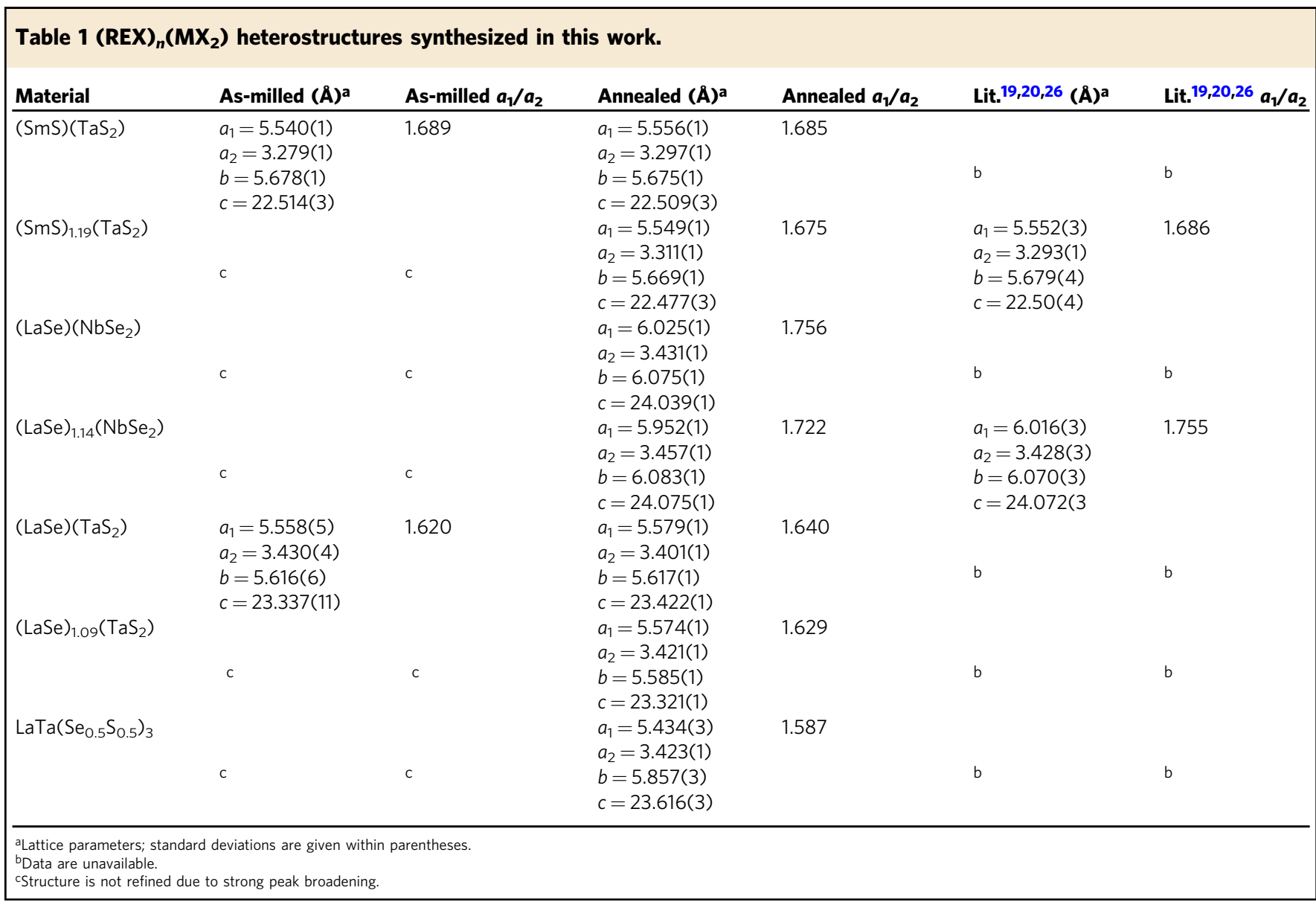

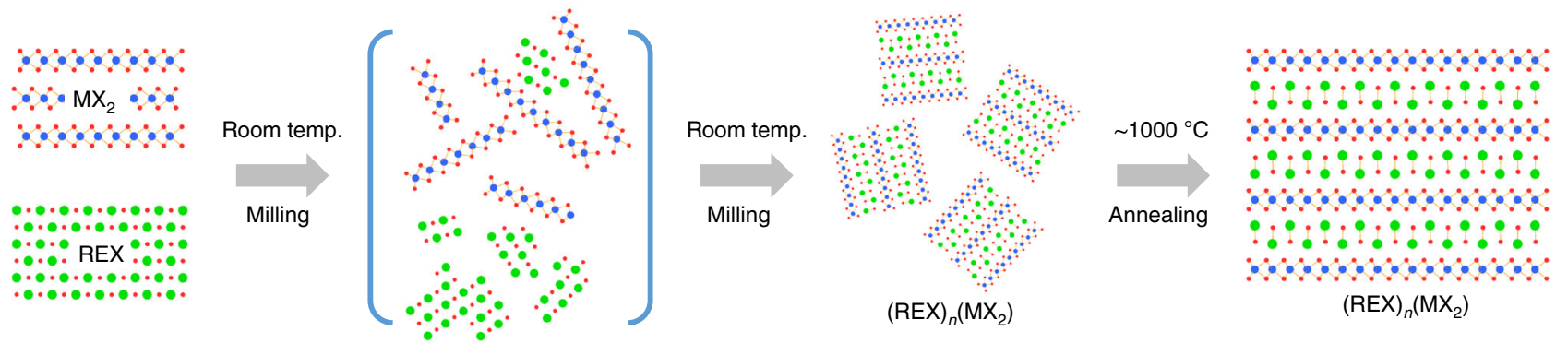

Fig. 3 Schematic representation of 3D heterostructures synthesis. Mechanochemical synthesis of $(R E X)_{n}\left(M X_{2}\right)$ heterostructures, where $R E=$ rare earth; $\mathrm{M}=\mathrm{Ta}$ or $\mathrm{Nb} ; \mathrm{X}=\mathrm{S}$ or $\mathrm{Se}$; and $n$ values vary between 1 and 1.2 .
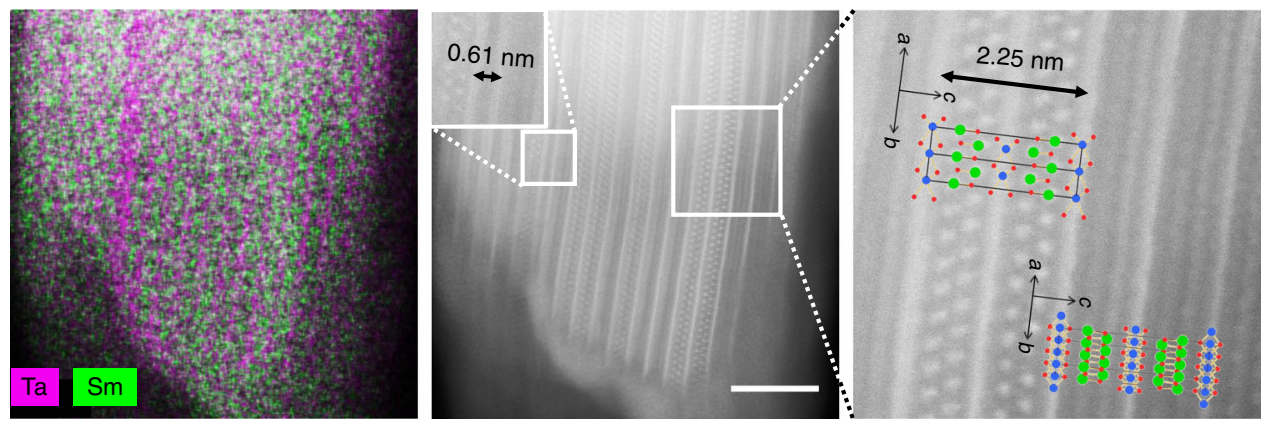

Fig. 4 Microscopy characterization of the $(\mathbf{S m S})\left(\mathbf{T a S}_{\mathbf{2}}\right)$. STEM-EDS and HAADF-STEM images of $(\mathrm{SmS})\left(\mathrm{TaS}_{2}\right)$ synthesized by ball milling and annealing of $\mathrm{SmS}$ and $\mathrm{TaS}_{2}$ mixture. A broad pink line in the EDS elemental mapping indicates a $\mathrm{TaS}_{2}-\mathrm{TaS}_{2}$ double layer that is clearly seen in the HAADF-STEM image (interlayer spacing $0.61 \mathrm{~nm}$ ). Scale bar, $5 \mathrm{~nm}$. 

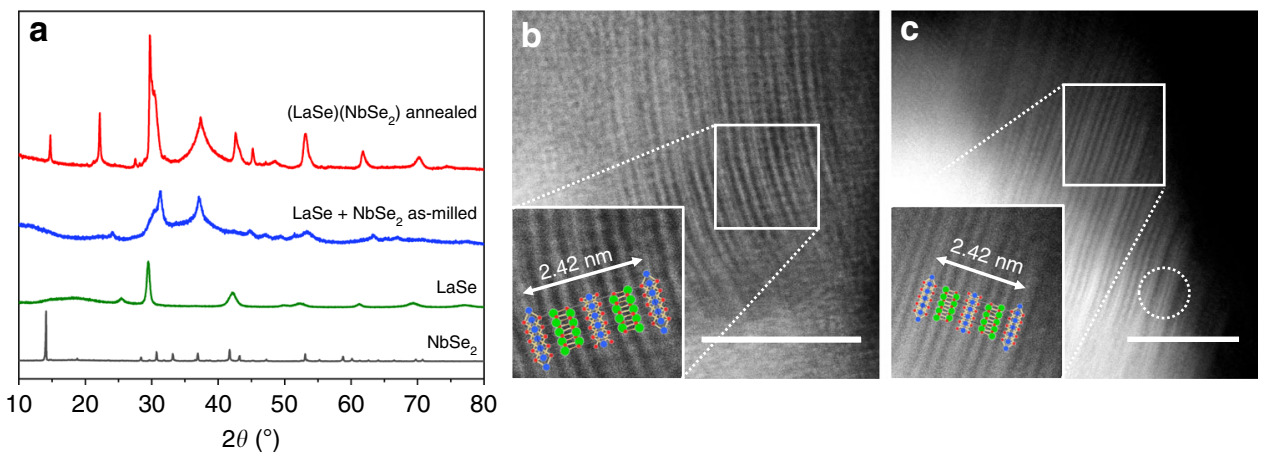

Fig. 5 Synthesis of the (LaSe)( $\mathbf{N b S e}_{\mathbf{2}}$ ) heterostructure. a PXRD patterns and $\mathbf{b}$ HAADF-STEM images of (LaSe)( $\left.\mathrm{NbSe}_{2}\right)$ obtained by ball milling LaSe and $\mathrm{NbSe}_{2}$ for $12 \mathrm{~h}$; $\mathbf{c}$ the HAADF-STEM image of the annealed material. An $\mathrm{NbSe}_{2}-\mathrm{NbSe}_{2}$ double layer is recognized in the lower right-hand corner of the image. Insets: enhanced atomic-scale views of 3D heterostructural arrangements. Scale bars, $5 \mathrm{~nm}$.

The results are quite remarkable as ball milling of elemental $\mathrm{Ta}$ and $\mathrm{S}$ does not produce detectable amounts of $\mathrm{TaS}_{2}$ even after prolonged processing (Supplementary Fig. 6). Therefore, it may be anticipated that SmS, mechanochemically generated at the early stages of the reaction, serves as a "scavenger" for $\left\{\mathrm{TaS}_{x}\right\}$ species, which form on the surface of the metallic Ta that is exposed to sulfur during mechanical processing. Alternatively, SmS may serve as a template for converting $\left\{\mathrm{TaS}_{x}\right\}$ into $\mathrm{TaS}_{2}$ layers of the thermodynamically stable $(\mathrm{SmS})_{n}\left(\mathrm{TaS}_{2}\right)$ heterostructure (Supplementary Fig. 7).

Very similar mechanochemical transformations are observed when an equimolar (1:1 molar ratio) mixture of $\mathrm{LaSe}$ and $\mathrm{NbSe}_{2}$ is ball milled in a shaker mill for up to $12 \mathrm{~h}$. In this case, the asmilled powder consists of disordered (LaSe) $\left(\mathrm{NbSe}_{2}\right)$ fragments, which further align upon annealing (Fig. 5b, c). Further structural analysis of the annealed $(\mathrm{LaSe})\left(\mathrm{NbSe}_{2}\right)$ reveals that it is isostructural to $(\mathrm{PbSe})_{1.10} \mathrm{NbSe}_{2}{ }^{25}$ and its lattice parameters (Table 1) agree well with those previously reported for $(\mathrm{LaSe})_{1.14}\left(\mathrm{NbSe}_{2}\right)^{20}$.

An attempt to mechanochemically prepare $(\mathrm{LaSe})_{1.14}\left(\mathrm{NbSe}_{2}\right)$ by combining LaSe and $\mathrm{NbSe}_{2}$, taken in the 1.14:1 molar ratio, produced a powder material that further crystalizes upon heating. Similar to $(\mathrm{SmS})_{1.19}\left(\mathrm{TaS}_{2}\right)$, the annealed $(\mathrm{LaSe})_{1.14}\left(\mathrm{NbSe}_{2}\right)$ also contains a minor secondary phase that does not belong to the precursor materials (Supplementary Fig. 8).

To explore further the scope of the mechanochemically facilitated 3D heterostructuring, we applied the newly developed synthetic approach to the synthesis of $(\mathrm{LaSe})_{n}\left(\mathrm{TaS}_{2}\right)$, where sulfur and selenium are expected to occupy different sites in adjacent $\mathrm{TaS}_{2}$ and LaSe sub-lattices. The PXRD patterns of both as-milled and annealed ( $\mathrm{LaSe})\left(\mathrm{TaS}_{2}\right)$ samples prepared from equimolar amounts of LaSe and $\mathrm{TaS}_{2}$ indicate the formation of 3D heterostructures (Supplementary Fig. 9) with lattice parameters falling between those of $(\mathrm{LaSe})\left(\mathrm{NbSe}_{2}\right)$ and $(\mathrm{SmS})\left(\mathrm{TaS}_{2}\right)$ (Table 1). Even after 10 days of holding at $1000^{\circ} \mathrm{C}$, the positions of Bragg peaks of the annealed $(\mathrm{LaSe})\left(\mathrm{TaS}_{2}\right)$ material remain unchanged (Supplementary Fig. 10).

Subsequently, the sample with the theoretical misfit stoichiometry, $(\mathrm{LaSe})_{1.09}\left(\mathrm{TaS}_{2}\right)$, was also prepared from LaSe and $\mathrm{TaS}_{2}$ combined in the 1.09:1 molar ratio. Also in this case, the lattice parameters of the annealed $(\mathrm{LaSe})_{1.09}\left(\mathrm{TaS}_{2}\right)$ are very close to those of $(\mathrm{LaSe})\left(\mathrm{TaS}_{2}\right)$ (Table 1) and a minor secondary phase impurity is found in the material (Supplementary Fig. 11a-c).

Unfortunately, STEM-EDS, Raman, and solid-state nuclear magnetic resonance (SSNMR) spectroscopies were unable to confirm unambiguously the exact locations of $\mathrm{S}$ and Se in the crystal lattice of $(\mathrm{LaSe})_{n}\left(\mathrm{TaS}_{2}\right)$. Therefore, we synthesized a LaTa $\left(\mathrm{Se}_{0.5} \mathrm{~S}_{0.5}\right)_{3}$ misfit material with a statistical distribution of $\mathrm{S}$ and Se by reacting elemental $\mathrm{La}, \mathrm{S}, \mathrm{Se}$, and $\mathrm{Ta}$, taken in 1:1:1.5:1.5 atomic ratio, using processing conditions identical to those developed during the preparation of $(\mathrm{SmS})\left(\mathrm{TaS}_{2}\right)$ from elements (Table 1 and Supplementary Fig. 11d-f).

The lattice parameters of the annealed $\mathrm{LaTa}\left(\mathrm{Se}_{0.5} \mathrm{~S}_{0.5}\right)_{3}$, especially the a parameter corresponding to the LaX sublattice, considerably deviate from those of $(\mathrm{LaSe})\left(\mathrm{TaS}_{2}\right)$, suggesting different chalcogen occupancies in $\mathrm{LaTa}\left(\mathrm{Se}_{0.5} \mathrm{~S}_{0.5}\right)_{3}$ and $(\mathrm{LaSe})_{n}\left(\mathrm{TaS}_{2}\right)$ heterostructures, which may be explained by assuming the preferential location of Se in the LaX sub-lattice of $(\mathrm{LaSe})_{n}\left(\mathrm{TaS}_{2}\right)$. Remarkably, mixing of $\mathrm{La}, \mathrm{Ta}, \mathrm{S}$, and Se in the 1:1:1.5:1.5 proportion and heating them without milling at $1000^{\circ} \mathrm{C}$ for 3 days does not produce $\mathrm{LaTa}\left(\mathrm{Se}_{0.5} \mathrm{~S}_{0.5}\right)_{3}$ (Supplementary Fig. 12), thus confirming the critical role of milling in the synthetic process.

${ }^{77}$ Se solid-state NMR and Raman spectroscopic studies. Structural models derived from PXRD and STEM experiments were further explored using ${ }^{77} \mathrm{Se}$ SSNMR and Raman spectroscopy. The ${ }^{77} \mathrm{Se}$ SSNMR spectra of the Se-containing $(\mathrm{REX})_{n}\left(\mathrm{MX}_{2}\right)$ heterostructures and binary metal chalcogenides LaSe, $\mathrm{NbSe}_{2}$, and $\mathrm{TaSe}_{2}$ are shown in Supplementary Fig. 13. The relatively narrow signal in the spectrum of LaSe is consistent with its highly symmetric cubic structure, where each Se is surrounded by six La atoms and the isotropic ${ }^{77} \mathrm{Se}$ chemical shift of ca. $500 \mathrm{p}$. p.m. is within the range of chemical shifts previously reported for metal selenides ${ }^{28}$. The ${ }^{77} \mathrm{Se}$ SSNMR spectra of both $\mathrm{NbSe}_{2}$ and $\mathrm{TaSe}_{2}$ exhibit broad axially symmetric powder patterns and their isotropic chemical shifts are in a much more positive range than that of LaSe (Supplementary Fig. 13a) or other semiconducting and insulating metal selenides ${ }^{28}$. The ${ }^{77} \mathrm{Se}$ SSNMR spectrum of commercial $\mathrm{NbSe}_{2}$ matches that previously reported ${ }^{29}$. Borsa et al..$^{30}$ attributed the large positive ${ }^{77} \mathrm{Se}$ isotropic shift of $\mathrm{NbSe}_{2}$ to a Knight shift, while Smith and co-workers ${ }^{29}$ attributed the positive ${ }^{77} \mathrm{Se}$ isotropic shift to the hyperfine coupling between $\mathrm{Se}$ and unpaired $d$ electrons residing on $\mathrm{Nb}(\mathrm{IV})$ atom ${ }^{29}$. The significant hyperfine shift anisotropy/Knight shift anisotropy/chemical shift anisotropy of ca. 2000 p.p.m. observed for both $\mathrm{NbSe}_{2}$ and $\mathrm{TaSe}_{2}$ is also consistent with the trigonal pyramidal coordination of selenium atoms in these layered TMDCs.

The ${ }^{77} \mathrm{Se}$ SSNMR spectra (Supplementary Fig. 13b) of $(\mathrm{LaSe})_{n}\left(\mathrm{NbSe}_{2}\right), \quad(\mathrm{LaSe})_{n}\left(\mathrm{TaS}_{2}\right)$, and $\mathrm{LaTa}\left(\mathrm{S}_{0.5} \mathrm{Se}_{0.5}\right)_{3}$ show no signals corresponding to the binary TMDCs, thus confirming complete conversion of the starting materials to products. The disappearance of the large positive shifts from the ${ }^{77}$ Se SSNMR spectra of the misfit heterostructures can be attributed to the electron transfer between their REX and $\mathrm{MX}_{2}$ sub-lattices, which is also in line with the results of the DFT calculations presented below. The ${ }^{77} \mathrm{Se}$ SSNMR spectra of the $(\mathrm{LaSe})_{n}\left(\mathrm{NbSe}_{2}\right)$ materials 
show anisotropic shielding patterns with breadths similar to those observed for the pure starting compounds. Se atoms in these heterostructures are situated in both $\mathrm{Nb}$ and La sub-lattices, and, as the symmetry of $\mathrm{Se}$ atoms in the $\mathrm{NbSe}_{2}$ layers of the misfit material remains largely unchanged, the magnitude of the shielding anisotropy is expected to be similar to that observed in the pure TMDC. While the ${ }^{77}$ Se SSNMR spectrum of LaSe produces a relatively narrow isotropic signal, the symmetry of Se atoms is reduced (Fig. 1) in the misfit heterostructures, leading to an anisotropic signal broadening. Consequently, it is challenging to assign definitively the ${ }^{77}$ Se SSNMR signals and to quantify the Se occupancy in the different sub-lattices by NMR spectroscopy (Supplementary Fig. 13).

Raman spectroscopy also confirms the absence of starting materials in all as-milled and annealed samples. Five characteristic bands are observed in the spectrum of $(\mathrm{SmS})\left(\mathrm{TaS}_{2}\right)$ (Supplementary Fig. 14a) with the bands at 119 and $148 \mathrm{~cm}^{-1}$ corresponding to intralayer vibrations of the SmS sub-lattice. The higher energy bands at 302 and $327 \mathrm{~cm}^{-1}$ can be assigned to the intralayer $E$ vibrational modes of the hexagonal $\mathrm{TaS}_{2}$ sub-lattice that are upshifted compared to the bulk $\mathrm{TaS}_{2}$. The band at $397 \mathrm{~cm}$

-1 is probably associated with intralayer $A$ vibrations in $\mathrm{TaS}_{2}$, which, in contrast to the $E$ mode, appear at the same position as $A_{1 g}$ vibrations in the pristine $\mathrm{TaS}_{2}{ }^{31,32}$. Similar peaks, shifted toward the lower wavenumbers, are also observed in the spectra of $(\mathrm{LaSe})\left(\mathrm{NbSe}_{2}\right)$ and $(\mathrm{LaSe})\left(\mathrm{TaS}_{2}\right)$ (Supplementary Fig. 14b, c). In all studied cases, the Raman spectra of the materials with the compositions $(\mathrm{REX})_{n}\left(\mathrm{MX}_{2}\right)$ (Supplementary Fig. 14d-f) are very similar to those of the respective $(\mathrm{REX})\left(\mathrm{MX}_{2}\right)$ heterostructures, which is in line with the results of our PXRD and STEM data.

DFT calculations. DFT calculations were used to assess stabilities of $(\mathrm{REX})_{n}\left(\mathrm{MX}_{2}\right)$ heterostructures with limiting nominal compositions $(n=1.14$ for $\mathrm{RE}=\mathrm{La}, \mathrm{M}=\mathrm{Nb}, \mathrm{X}=\mathrm{Se}$, and $n=1.19$ for $\mathrm{RE}=\mathrm{Sm}, \mathrm{M}=\mathrm{Ta}$, and $\mathrm{X}=\mathrm{S}$ ). Calculation details are included into Supplementary Note 1 . The total energy $\left(E_{\text {tot }}\right)$ and formation energy $\left(E_{\text {form }}\right)$ for each phase are listed in Table 2 , as well as relative energies $\Delta E$ to highlight the relative stability of the heterostructures. Recall: $E_{\text {form }}$ is the total energy relative to the weighted probabilities of the constituent-elemental energies; it is the energy gained [or lost] in the formation of the heterostructure, as measured in calorimetry, for example, $E_{\text {tot }}$ is the energy of the heterostructure relative to atomic vacuum.

The relative energy $\Delta E$ (per atom) for cubic-(REX) and $(\mathrm{REX})_{n}\left(\mathrm{MX}_{2}\right)$ with respect to $2 \mathrm{H}-\mathrm{MX}_{2}$ is plotted in Fig. 6. The relative energies (total energies $\left.E_{\text {tot }}\right)$ of $(\mathrm{SmS})_{1.19}\left(\mathrm{TaS}_{2}\right)$ and $(\mathrm{LaSe})_{1.14}\left(\mathrm{NbSe}_{2}\right)$ are $-0.163 \mathrm{eV} /$ atom $(-1.578 \mathrm{eV} /$ atom $)$ and $-0.021 \mathrm{eV} /$ atom $(-1.486 \mathrm{eV} /$ atom), respectively, see Table 2 . $\Delta E$ s are significantly lower than their binary precursors, that is, at $-0.922 \mathrm{eV} /$ atom below $\mathrm{SmS}$ and $-0.163 \mathrm{eV} /$ atom below $\mathrm{TaS}_{2}$ for $(\mathrm{SmS})_{1.19}\left(\mathrm{TaS}_{2}\right)$, and, similarly, at $-0.406 \mathrm{eV} /$ atom below LaSe and $-0.021 \mathrm{eV} /$ atom below $\mathrm{NbSe}_{2}$ for $(\mathrm{LaSe})_{1.14}\left(\mathrm{NbSe}_{2}\right)$. This relative energy shows that mixing of $\mathrm{MX}_{2}$ and REX lowers the total energy of the misfit compounds, and the negative $E_{\text {form }}$ of $(\mathrm{SmS})_{1.19}\left(\mathrm{TaS}_{2}\right)$ and $(\mathrm{LaSe})_{1.14}\left(\mathrm{NbSe}_{2}\right)$ in Table 2 further shows the increased stability of misfit compounds. $(\mathrm{REX})_{n}\left(\mathrm{MX}_{2}\right)$ heterostructures are layered $\mathrm{MX}_{2}$ materials intercalated by REX compounds, where metal atoms $(\mathrm{M})$ adopt a +4-oxidation state, $\mathrm{RE}$ elements assume a +2 -oxidation state, and chalcogens (X) have -2-oxidation state. As it intercalates, their structure can be stabilized by electron transfer between the REX fragment and the $\mathrm{MX}_{2}$ network ${ }^{33}$. $(\mathrm{SmS})_{1.19}\left(\mathrm{TaS}_{2}\right)$ and $(\mathrm{LaSe})_{1.14}\left(\mathrm{NbSe}_{2}\right)$ represent good examples of such incommensurate misfit materials, and their electronic structures will be discussed as two representative cases of S- and Se-containing heterostructures.

The density of states (DOS) of binary precursors, SmS and $\mathrm{TaS}_{2}$, is shown in Fig. 7a, b. The sulfur $s$-bands in $\mathrm{SmS}$ are far below the Fermi energy $\left(E_{\mathrm{F}}\right)$, separated from the other valence states by $7.0 \mathrm{eV}$. The bands near $E_{\mathrm{F}}$ mainly consist of sulfur $p$ bands that are weakly hybridized with $\mathrm{Sm} d$-orbitals. The weak or lack of hybridization between $\mathrm{Sm} s$ - and $d$-bands and S p-orbitals is evident from non-overlapping (spherical) charge density of Sm and $\mathrm{S}$ atoms, suggesting dominance of ionic bonding in SmS.

In contrast, the DOS of $2 \mathrm{H}-\mathrm{TaS}_{2}$ (Fig. 7b) shows two separate sets of energy bands near $\mathrm{E}_{\mathrm{F}}$ with the energy width of $\sim 1 \mathrm{eV}$,

Table 2 Total $\left(E_{\text {total }}\right)$ and formation $\left(E_{\text {form }}\right)$ energies of $(\operatorname{REX})_{n}\left(M X_{2}\right)$ heterostructures with comparison to cubic-REX and $2 H-$ $\mathrm{MX}_{2}$ chalcogenides ${ }^{\mathrm{a}}$.

\begin{tabular}{|c|c|c|c|c|c|c|c|}
\hline System & $E_{\text {total }}(\mathrm{eV} /$ atom $)$ & $\Delta E(\mathrm{eV} /$ atom $)$ & $E_{\text {form }}(\mathrm{eV} /$ atom $)$ & System & $E_{\text {total }}(\mathrm{eV} /$ atom $)$ & $\Delta E(e V /$ atom $)$ & $E_{\text {form }}(\mathrm{eV} /$ atom) \\
\hline Cubic-SmS & -6.4487 & +0.759 & -2.0248 & Cubic-LaSe & -6.2077 & +0.385 & -1.9948 \\
\hline$(\mathrm{SmS})_{1.19}\left(\mathrm{TaS}_{2}\right)$ & -7.3708 & -0.163 & -1.5781 & $(\mathrm{LaSe})_{1.14}\left(\mathrm{NbSe}_{2}\right)$ & -6.6141 & -0.021 & -1.48570 \\
\hline
\end{tabular}
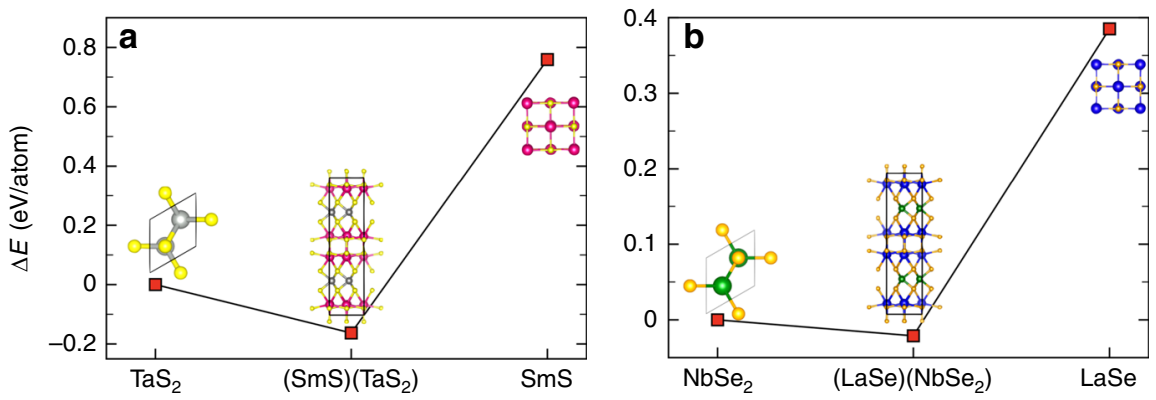

Fig. 6 DFT calculations for $(\mathbf{R E X})_{\mathbf{n}}\left(\mathbf{M X}_{\mathbf{2}}\right)$ heterostructures. a Relative DFT energies $\Delta E$ (i.e., the difference of total energies per atom relative to $2 \mathrm{H}-\mathrm{MX} \mathrm{X}_{2}$ ) of $(\mathrm{SmS})_{n}\left(\mathrm{TaS}_{2}\right), n=1.19$ and of $\mathbf{b}\left(\mathrm{LaSe}_{n}\left(\mathrm{NbSe}_{2}\right), n=1.14\right.$ misfit compounds compared to single-phase cubic-REX [SmS, LaSe] and 2H-MX ${ }_{2}$ [TaS ${ }_{2}$, $\mathrm{NbSe}_{2}$ ] building blocks. 

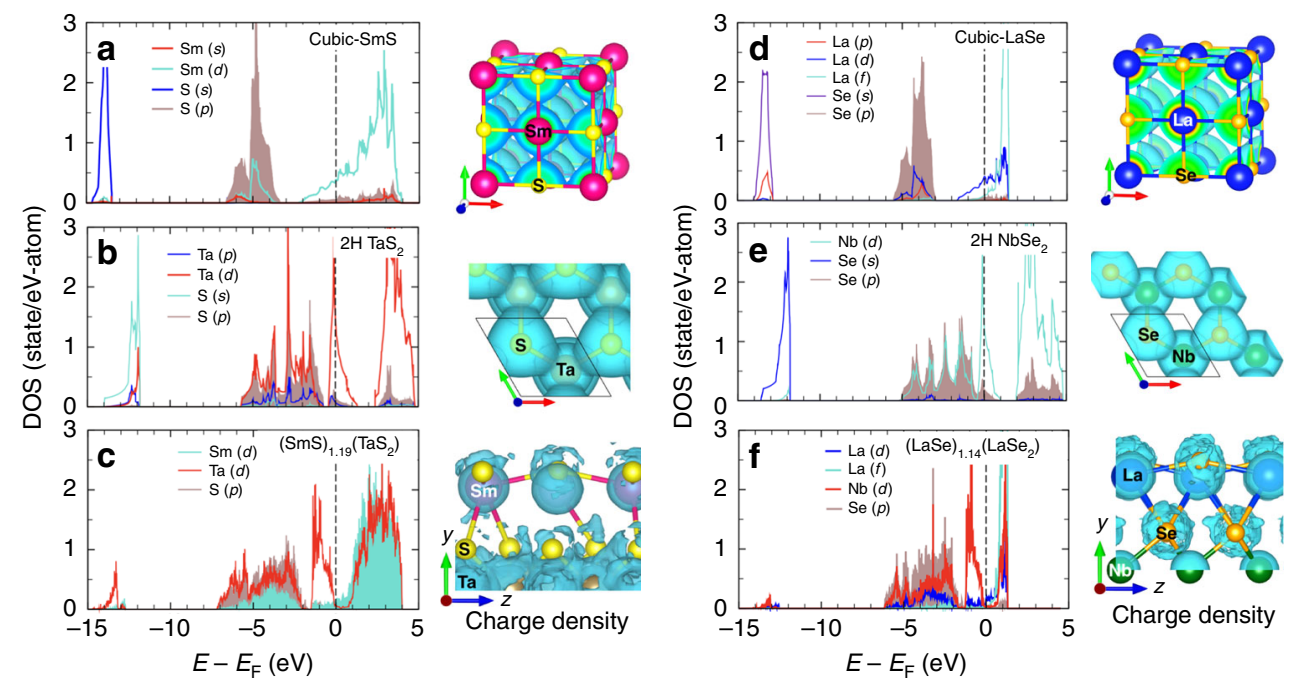

Fig. 7 DOS calculations for $(\mathbf{R E X})_{\mathbf{n}}\left(\mathbf{M X}_{\mathbf{2}}\right)$ heterostructures. a Partial DOS and charge density of cubic-SmS, b $2 \mathrm{H}-\mathrm{TaS}{ }_{2}, \mathbf{c}\left(\mathrm{SmS}_{1.19}\left(\mathrm{TaS}_{2}\right)\right.$, d cubic-LaSe, e $2 \mathrm{H}-\mathrm{NbSe}_{2}$, and $\mathbf{f}\left(\mathrm{LaSe}_{1.14}\left(\mathrm{NbSe}_{2}\right)\right.$. Charge density isosurfaces $\left(0.03 \mathrm{e}^{-} / \AA^{3}\right)$ are plotted to highlight the distribution.

which are formed by the crystal field created by octahedrally (prismatic) arranged S around Ta. The DOS associated with Ta $d$ band shows a strong maximum near $E_{\mathrm{F}}$ and the bands just below $E_{\mathrm{F}}$ consist of the $\mathrm{S} p$-orbitals with a non-negligible contribution from Ta $d$-orbitals, whereas the lowest-energy bands consist of $S$ $s$-orbitals and are located at $-12.5 \mathrm{eV}$.

In $(\mathrm{SmS})_{1.19}\left(\mathrm{TaS}_{2}\right)$, the $\mathrm{S} p$-bands and $\mathrm{Sm} d$-bands are shifted compared to those in the parent $2 \mathrm{H}-\mathrm{TaS}_{2}$ and cubic-SmS materials (Fig. 7c). A fraction of their shift can be attributed to higher filling of $d_{z^{2}}$-state in Ta, while other components of the shift occur by lowering the energy of $S$ s-orbitals due to Coulombic interactions with $\mathrm{Sm}$. The sulfur $s$-band contributed by the $\mathrm{SmS}$ fragment is shifted toward lower energies (near -16.5 $\mathrm{eV}$ below $E_{\mathrm{F}}$ ) with respect to the parent cubic-SmS, most likely due to the presence of an electron in the Sm $d$-band. The set of bands between $E_{\mathrm{F}}$ and $-1.5 \mathrm{eV}$ corresponds to $d_{z^{2}}$-states in Ta, whose bandwidth is similar to that in the parent $\mathrm{TaS}_{2}$. The lower edge of the $d_{z^{2}}$-band in $(\mathrm{SmS})_{1.19}\left(\mathrm{TaS}_{2}\right)$ is at $0.5 \mathrm{eV}$ below $E_{\mathrm{F}}$, while it crosses $E_{\mathrm{F}}$ in $2 \mathrm{H}-\mathrm{TaS}_{2}$. Thus, the $d_{z^{2}}$-band in $(\mathrm{SmS})_{1.19}\left(\mathrm{TaS}_{2}\right)$ is partially occupied with electron density transferred from SmS to the $\mathrm{TaS}_{2}$ sub-lattice. In binary $\mathrm{TaS}_{2}$, the $\mathrm{Ta} d_{z^{2}}$-band is half filled (i.e., one hole per $\mathrm{Ta}$ atom). However, a Bader charge analysis ${ }^{34}$ of $(\mathrm{SmS})_{1.19}\left(\mathrm{TaS}_{2}\right)$ (Supplementary Table 3 and Supplementary Figs. 15 and 16) reveals that, in fact, the Ta $d_{z^{2}}$-band contains only $0.17-0.21$ holes per Ta, indicating a charge transfer of $0.83-0.79$ from cubic-SmS to $2 \mathrm{H}$ $\mathrm{TaS}_{2}$. The charge density isocontour $\left(0.03 e^{-} / \AA^{3}\right)$ is shown in Fig. $7 \mathrm{c}$ and highlights the interlayer coupling in $(\mathrm{SmS})_{1.19}\left(\mathrm{TaS}_{2}\right)$ mediated by this charge transfer. The charge densities are shown between $-2 \mathrm{eV}$ to $E_{\mathrm{F}}$. They indicate a significant charge density overlap along Sm-S-Ta bonding directions via the $S$ atom that belongs to the $\mathrm{TaS}_{2}$ layer. The Bader analysis further confirms that charge transfer can stabilize the incommensurate $(\mathrm{REX})_{n}\left(\mathrm{MX}_{2}\right)$ systems (Supplementary Table 3 and Supplementary Figs. 15 and 16).

The electronic structures of cubic-LaSe, $2 \mathrm{H}-\mathrm{NbSe}_{2}$, and $(\mathrm{LaSe})_{1.14}\left(\mathrm{NbSe}_{2}\right)$ are similar to $(\mathrm{SmS})_{1.19}\left(\mathrm{TaS}_{2}\right)$ and its building blocks, see Fig. 7d-f. The energy gap between Se $s$ - and $p$-bands in $(\mathrm{LaSe})_{1.14}\left(\mathrm{NbSe}_{2}\right)$ is $\sim 3.4 \mathrm{eV}$, smaller than the corresponding values for cubic-LaSe $(7.5 \mathrm{eV})$ and $2 \mathrm{H}-\mathrm{NbSe}_{2}(6.80 \mathrm{eV})$. In $(\mathrm{LaSe})_{1.14}\left(\mathrm{NbSe}_{2}\right)$, two bands at -6.5 and $-1.60 \mathrm{eV}$ are formed from Se $p$-bands and $d$-bands of $\mathrm{La}$ and $\mathrm{Nb}$, while the bands between -1.4 and $1.3 \mathrm{eV}$ originate from $\mathrm{Nb} d_{z^{2}}$-orbital that, in turn, is hybridized with Se $p$-states and La $d$-states. The $\mathrm{Nb}$ $d_{z^{2}}$-band contains $\sim 0.2$ holes per $\mathrm{Nb}$ atom, indicating a transfer of $\sim 0.8$ electrons from LaSe to $\mathrm{NbSe}_{2}$. In this case, charge transfer proceeds through Se atom and serves as a "glue" for the entire hetero-assembly.

In summary, DFT calculations confirm that charge transfer from REX to $\mathrm{MX}_{2}$ stabilizes (REX) ${ }_{n}\left(\mathrm{MX}_{2}\right)$ misfit compounds, and glues together the structurally different elements of such heterostructures. The directionality of the interlayer charge densities suggests that the bonding character is largely covalent in misfit compounds and the effective charge sharing increases the bonding strength, which lowers the free energy of misfit materials with respect to binary REX and $\mathrm{MX}_{2}$ phases. This helps to understand the formation of the (REX $)_{n}\left(\mathrm{MX}_{2}\right)$ heterostructures from both binary metal chalcogenides and the elements, for $(\mathrm{REX})_{n}\left(\mathrm{MX}_{2}\right)$ is the most thermodynamically stable atomic arrangement in the studied RE-M-3X systems. Finally, our calculations identify the chalcogen atoms as conduit for the charge transfer in $(\mathrm{REX})_{n}\left(\mathrm{MX}_{2}\right)$-a remarkable result not reported before, to the best of our knowledge.

Electronic transport studies. Investigations of the electronic transport in $(\mathrm{REX})_{n}\left(\mathrm{MX}_{2}\right)$ prepared in this work provide further valuable information about their physical and chemical nature and serve as an additional marker for consistency of the developed synthetic approach.

The temperature dependencies of the electrical resistivities, $\rho$ (T), for as-milled (LaSe) $\left(\mathrm{NbSe}_{2}\right)$ and $(\mathrm{SmS})\left(\mathrm{TaS}_{2}\right)$, measured between 300 and $2 \mathrm{~K}$ in the absence of external magnetic field ( $H$ $=0$ ), are shown in Fig. 8. The resistivity values observed are between $0.12 \Omega-\mathrm{cm}(\mathrm{LaSe})\left(\mathrm{NbSe}_{2}\right)$ and $0.074 \Omega-\mathrm{cm}(\mathrm{SmS})\left(\mathrm{TaS}_{2}\right)$ at $300 \mathrm{~K}$. They slowly increase with decreasing temperature and reach $0.45 \Omega$-cm for $(\mathrm{LaSe})\left(\mathrm{NbSe}_{2}\right)$ at $50 \mathrm{~K}$ and $0.18 \Omega-\mathrm{cm}$ for $(\mathrm{SmS})\left(\mathrm{TaS}_{2}\right)$ at $30 \mathrm{~K}$.

Around $30-50 \mathrm{~K}$, the resistivities of both as-milled materials begin to grow exponentially, indicating possible transition from semiconducting to insulating states that has not been reported for misfit heterostructures in the past and is not observed in the annealed samples synthesized in this work. The nature of the discovered phenomenon is quite intriguing and requires additional studies, which will be published elsewhere.

The annealed $(\mathrm{LaSe})\left(\mathrm{NbSe}_{2}\right)$ and $(\mathrm{SmS})\left(\mathrm{TaS}_{2}\right)$ demonstrate metallic behavior with electrical resistivities of $9.5 \mathrm{~m} \Omega-\mathrm{cm}$ for 

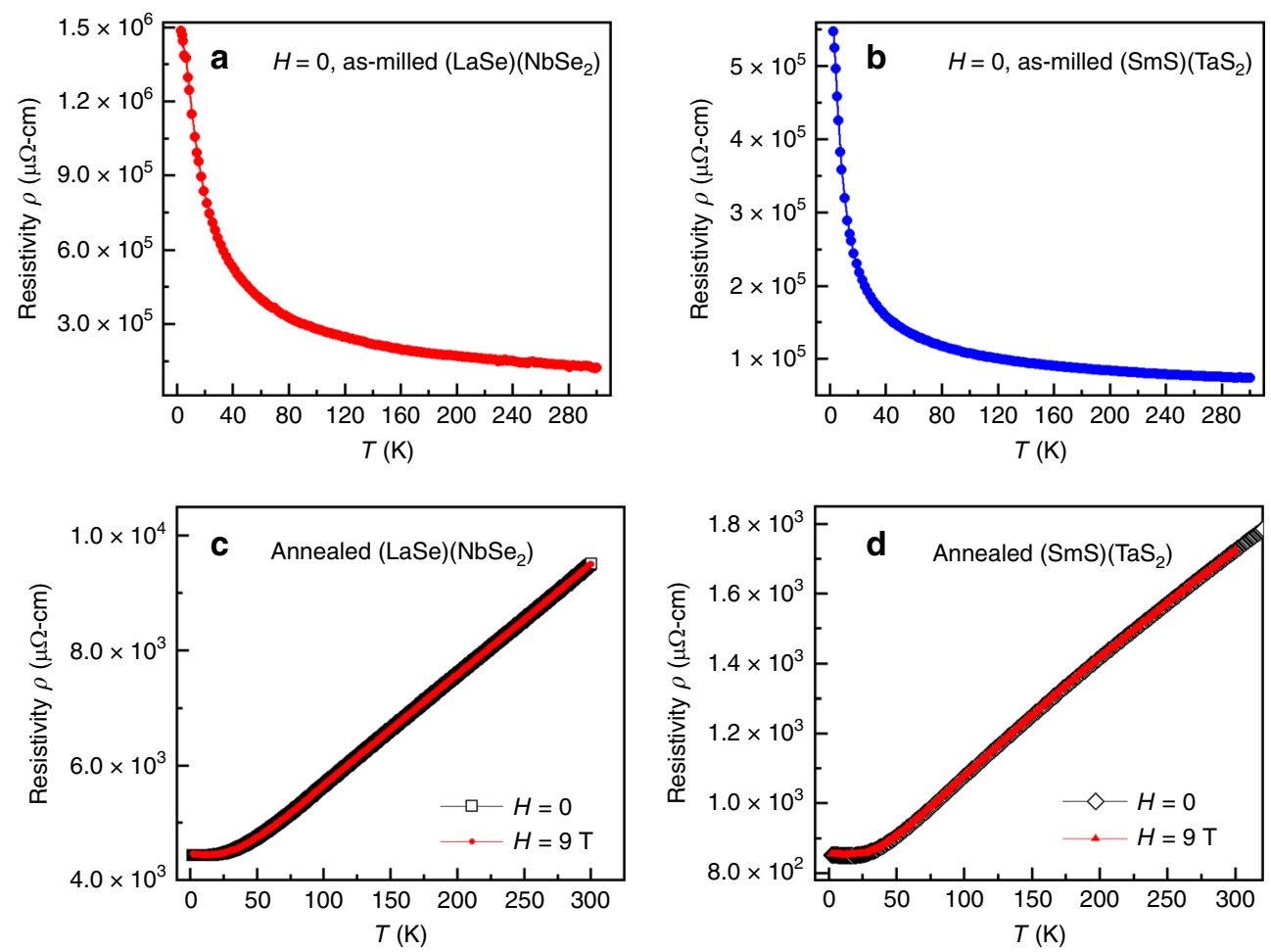

Fig. 8 Electrical resistivity characterization of heterostructures. a Electrical resistivity versus temperature measured in 0 and $9 \mathrm{~T}$ fields for as-milled and $\mathbf{c}$ annealed $(\mathrm{LaSe})\left(\mathrm{NbSe}_{2}\right) ; \mathbf{b}$ as-milled and $\mathbf{d}$ annealed $(\mathrm{SmS})\left(\mathrm{TaS}_{2}\right)$.

(LaSe) $\left(\mathrm{NbSe}_{2}\right)$ and $1.7 \mathrm{~m} \Omega$-cm for $(\mathrm{SmS})\left(\mathrm{TaS}_{2}\right)$ at $300 \mathrm{~K}$, that is, $\sim 10$ and 40 times lower values than those observed for as-milled samples. They further decrease with decreasing temperature and reach $4.4 \mathrm{~m} \Omega-\mathrm{cm}$ for $(\mathrm{LaSe})\left(\mathrm{NbSe}_{2}\right)$ at $12 \mathrm{~K}$ and $0.8 \mathrm{~m} \Omega-\mathrm{cm}$ for $(\mathrm{SmS})\left(\mathrm{TaS}_{2}\right)$ at $15 \mathrm{~K}$ (Fig. 8c, d). The annealed $(\mathrm{LaSe})_{1.14}\left(\mathrm{NbSe}_{2}\right)$ demonstrates transport behaviors similar to those of the annealed $(\mathrm{LaSe})\left(\mathrm{NbSe}_{2}\right)$ (Supplementary Fig. 17). The transport properties of the annealed $(\mathrm{SmS})\left(\mathrm{TaS}_{2}\right)$ material are in line with that previously reported for a single-crystal of $(\mathrm{SmS})_{1.19}\left(\mathrm{TaS}_{2}\right)^{21}$. Strong external magnetic field of $9 \mathrm{~T}$ does not show a measurable effect on the electronic transport of the investigated samples.

Both annealed $(\mathrm{LaSe})_{n}\left(\mathrm{TaS}_{2}\right)$ materials also display metallic behaviors that do not depend on their formal chemical composition (Supplementary Fig. 18) and resemble that of $(\mathrm{SmS})\left(\mathrm{TaS}_{2}\right)$ and $(\mathrm{LaSe})\left(\mathrm{NbSe}_{2}\right)$. However, unlike the latter, $(\mathrm{LaSe})_{n}\left(\mathrm{TaS}_{2}\right)$ exhibit distinct resistivity drops $\sim 4 \mathrm{~K}$ that coincide with superconducting transitions in metallic $\mathrm{Ta}^{35,36}$ and $\mathrm{TaS}_{1}$ ${ }_{x} \mathrm{Se}_{x}$ with low Se content, $x<0.2^{37}$-trace impurities that may have formed during the heat treatment. Thus, electronic transport of all studied annealed materials shows remarkably little dependence on their formal chemical compositions and is in line with data reported for $(\mathrm{REX})_{n}\left(\mathrm{MX}_{2}\right)$ with $n>1$.

\section{Discussion}

In conclusion, we demonstrate that mechanical milling, in contrast to common belief about its destructive nature, consistently and reproducibly facilitates $3 \mathrm{D}$ heterostructural formation via disassembly and re-ordering of structurally dissimilar RE and transition-metal chalcogenides at ambient temperature and pressure. The created misfit moieties can further structurally align under heat treatment in a way that is common for molecular solids and polymers, that is, without altering their intrinsic atomic arrangements. Distinct from conventional heterostructuring techniques, the discovered synthetic approach may enable the $3 \mathrm{D}$ heterostructures with site-separated components that are inaccessible via conventional synthetic routes.

Our DFT calculations uncovered unique details about chemical bonding and charge transfer in the incommensurate misfit metal chalcogenides that allows understanding the mechanochemical formation from binary metal chalcogenides or pure elements.

Detailed investigation of the chemical structures and properties of the mechanochemically synthesized misfit heterostructures uncovered a clear contradiction between anticipated stoichiometries of the $(\mathrm{REX})_{n}\left(\mathrm{MX}_{2}\right)$ solids and their experimentally achieved chemical compositions, which can be extended onto other misfit materials reported in the literature.

\section{Methods}

Materials. Ultra-high-purity $\mathrm{Ar}$ (Matheson, 99.999\%), ultra-high-purity $\mathrm{He}$ (Matheson, 99.999\%), $\mathrm{NbSe}_{2}$ (Alfa Aesar, 99.8\% purity), La (Materials Preparation Center, Ames Laboratory [MPC]), 99.99\% REE purity), Sm (MPC, REE 99.99\% purity), S (Alfa Aesar, 99.9995\% purity), and Se (Alfa Aesar, 99.999\% purity). $\mathrm{TaS}_{2}$, SmS, and LaSe were prepared in-house from the elements.

Synthesis. In a typical experiment (Fig. 3), a $2 \mathrm{~g}$ sample of the physical mixture of a TMDC and a RE chalcogenide (or a RE metal and a chalcogen), taken in stoichiometric proportions, was milled either in a silicon nitride milling container together with three $12.7 \mathrm{~mm}$ silicon nitride balls using a shaker mill (SPEX 8000M), or in a zirconia vial with five $15 \mathrm{~mm}$ zirconia balls using a two-station horizontal planetary mill (Fritsch, Pulverisette 7) for periods of time between 12 and $30 \mathrm{~h}$ Materials handling, including loading of the vial, was performed under ultra-highpurity argon in a glove box. After ball milling, the obtained material was pressed into a pellet under argon in a glove box, placed in a quartz tube, sealed under 0.75 bar of ultra-high-purity helium, and heat treated by ramping the temperature to $1000{ }^{\circ} \mathrm{C}$ and annealing the material for 3 days. Thereafter, the sample was cooled down with the furnace to room temperature. For characterization, the prepared solid was crushed in an agate mortar with an agate pestle and stored in a glove box, both operations were performed under high-purity argon.

TMDC and RE chalcogenide precursors: $\mathrm{NbSe}_{2}$ was used as received. $\mathrm{TaS}_{2}$ was prepared by ball milling of a nearly stoichiometric mixture of tantalum and sulfur (5\% excess) for $4 \mathrm{~h}$ in a shaker mill in a silicon nitride container followed by a 3 day heat treatment in a quartz tube sealed under 0.75 bar pressure of the ultra- 
high-purity helium. The PXRD analysis of the obtained material confirmed the formation of the pure $\mathrm{TaS}_{2}$ phase (Supplementary Fig. 1).

LaSe and SmS were prepared by ball milling of stoichiometric mixtures of RE metals and chalcogens for $12 \mathrm{~h}$ in a shaker mill in a silicon nitride container. The PXRD pattern contained only peaks corresponding to RE chalcogenides (Supplementary Fig. 2).

Powder X-ray diffraction. Phase analysis and structural characterization of the reaction products were carried out using PXRD at room temperature on a PANalytical X'Pert PRO diffractometer with $\mathrm{Cu}-\mathrm{K} \alpha$ radiation in the range of Bragg angles $5^{\circ} \leq 2 \theta \leq 80^{\circ}$. Lattice parameters were determined using Le Bail profile fitting (Supplementary Fig. 19) in FullProf ${ }^{38}$ with background approximated through linear interpolation between data points selected from regions free of Bragg reflections, and peak shapes approximated using pseudo-Voigt function. In the Le Bail refinements, RE chalcogenide REX (space group $F m 2 m$ ) and transition-metal chalcogenide $\mathrm{MX}_{2}$ (space group $\mathrm{Cm} 2 \mathrm{~m}$ ) sub-lattices were fitted as separate phases, each contributing its own set of Bragg reflections to the observed X-ray powder diffraction pattern. Since both commensurate axes $(b$ and $c$ ) are identical for the two sub-lattices, their values were constrained as such during the refinements. The misfit in the structure occurs along the $a$ direction; therefore, lattice parameters $a_{1}$ (for REX) and $a_{2}$ (for $\mathrm{MX}_{2}$ ) were refined independently for each of the sub-lattices.

Microscopy. TEM experiments were carried out on a Titan Themis (FEI) probe Cs-corrected TEM. HAADF-STEM images were acquired with a convergence semi-angle of $18 \mathrm{mrad}$ and a collection semi-angle of $99-200 \mathrm{mrad}$ at $200 \mathrm{kV}$. EDS analysis was performed using a Super-X EDS detector.

Raman spectroscopy. Powdered samples were spread on glass coverslips and analyzed with a Horiba XploRA Raman microscope (HORIBA Scientific, Edison, NJ) using 532-nm excitation $\left(8.3 \times 10^{3} \mathrm{~W} / \mathrm{cm}^{2}\right)$ and a $100 \times(0.90 \mathrm{NA})$ objective. The detector was a front-illuminated Horiba Synapse EMCCD camera, and the acquisition time was $60 \mathrm{~s}$. For each sample, the displayed spectrum was an average of 10 locations.

${ }^{77}$ Se solid-state NMR. ${ }^{77} \mathrm{Se}$ SSNMR experiments were performed on a $9.4 \mathrm{~T}$ wide bore magnet $\left(v_{0}(77 \mathrm{Se})=76.34 \mathrm{MHz}\right)$ equipped with a Bruker Avance III HD spectrometer. All spectra were obtained on an $\mathrm{H}-\mathrm{X}$ double resonance Bruker static probe with a 4-mm transverse coil. Samples were packed into 4-mm zirconia rotors. Static ${ }^{77}$ Se SSNMR spectra were obtained with the wideband, uniform rate, smooth truncation-Carr-Purcell Meiboom-Gill (WURST-CPMG) pulse sequence ${ }^{39-41}$. The WURST pulses were used for excitation and refocusing. The WURST pulses were $30 \mu$ s in duration and swept over a total frequency range of $600 \mathrm{kHz}( \pm 300 \mathrm{kHz}$ frequency offsets). This sweep width was sufficient to excite and refocus signals from paramagnetically shifted sites and cover the wide chemical shift range of ${ }^{77} \mathrm{Se}$. Spectra were obtained by processing the first full echo in the CPMG echo train. Transformation of all echoes in the CPMG echo train typically resulted in significant intensity distortions. The duration of the first spin echo was $4.5 \mathrm{~ms}$. Spectra were obtained with recycle delays of 0.5 or $1 \mathrm{~s}$, except for the $(\mathrm{LaSe})_{114}\left(\mathrm{NbSe}_{2}\right)$ as-milled sample for which a $60 \mathrm{~s}$ recycle delay was used. Between 4092 and 81,920 scans were acquired for each sample.

DFT calculations. Electronic structure of $(\mathrm{REX})_{n}\left(\mathrm{MX}_{2}\right)$ heterostructures are calculated using the Vienna Ab-Initio Simulation Package ${ }^{42,43}$. The valence interaction is described by projector augmented-wave method ${ }^{44}$ with energy cutoff of 350 $\mathrm{eV}$ for the plane-wave orbitals. The Monkhorst-Pack $k$-mesh of $1 \times 1 \times 2$ and $3 \times$ $3 \times 9$ is used for Brillouin zone integration during full relaxation (lattice + ionic) and charge self-consistency (including electronic DOS) ${ }^{45}$, respectively; Monkhorst-Pack $k$-mesh of $25 \times 25 \times 25$ and $19 \times 19 \times 11$ are used for REX and $\mathrm{MX}_{2}$, respectively. Total energies and forces are converged to $10^{-5} \mathrm{eV} /$ cell and 0.01 $\mathrm{eV} / \mathrm{A}$. The Perdew-Burke-Ernzerhof ${ }^{46}$ exchange-correlation functional is employed in the generalized gradient approximation. We also perform the Bader charge analysis ${ }^{34}$ to determine the local charge distributions after charge selfconsistency. The parent structures REX (SmS; LaSe) and $\mathrm{MX}_{2}\left(\mathrm{NbSe}_{2} ; \mathrm{TaS}_{2}\right)$ are adopted from the Materials Project ${ }^{47}$. We project $(\operatorname{REX})_{n}\left(\mathrm{MX}_{2}\right)$ unit cell along $(00$ 1) in the large supercell (see Supplementary Tables 1 and 2) to make the incommensurate crystal structure commensurate, and to simplify the calculation of misfit compounds.

Electrical resistivity measurements. The electrical resistivities $(\rho)$ have been measured in the absence of an external magnetic field and in the field of $9 \mathrm{~T}$ using a standard four-probe technique in the Physical Property Measurements System (Quantum Design, Inc.). The powdered as-milled samples are pressed with an 8ton hydraulic press, polished and cut into rectangular pieces with $4 \times 2 \times 1 \mathrm{~mm}^{3}$ dimensions. The annealed materials are mechanically robust and could be cut to the required dimensions without additional pressing.

\section{Data availability}

Data that support plots within this paper and other findings of this study are available from the corresponding author upon reasonable request.

Received: 26 November 2019; Accepted: 15 May 2020;

Published online: 12 June 2020

\section{References}

1. Geim, A. K. \& Grigorieva, I. V. Van der Waals heterostructures. Nature 499, 419-425 (2013).

2. Novoselov, K. S., Mishchenko, A., Carvalho, A. \& Castro Neto, A. H. 2D materials and Van der Waals heterostructures. Science 353, aac9439 (2016).

3. Pervov, V. S. \& Makhonina, E. V. Incommensurate suprastructures: new problems of inorganic solid-state chemistry. Russ. Chem. Rev. 69, 481-489 (2000).

4. Serra, M. \& Tenne, R. Nanotubes from misfit layered compounds. J. Coord. Chem. 71, 1669-1678 (2018).

5. Kolobov, A. \& Tominaga, J. Two-Dimensional Transition-Metal Dichalcogenides. Springer Series in Materials Science, 239 (Springer International Publishing AG, Switzerland, 2016).

6. Lacopi, F., Boeckl, J. J. \& Jagadish, C. Semiconductors and Semimetals, Vol. 95 2D Materials (Academic Press, Cambridge, San Diego, Oxford, London, 2016).

7. Cai, Z., Liu, B., Zou, X. \& Cheng, H.-M. Chemical vapor deposition growth and applications of two-dimensional materials and their heterostructures. Chem. Rev. 118, 6091-6133 (2018).

8. Li, H., Li, Y., Aljarb, A., Shi, Y. \& Li, L.-J. Epitaxial growth of two-dimensiona layered transition-metal dichalcogenides: growth mechanism, controllability, and scalability. Chem. Rev. 118, 6134-6150 (2018).

9. US Department of Energy. Synthesis and Processing Science https://science.osti. gov/bes/Research (2019)

10. Hlova, I. Z. et al. Multi-principal element transition metal dichalcogenides via reactive fusion of 3D-heterostructures. Chem. Commun. 54, 12574-12577 (2018).

11. Rouxel, J., Meerschaut, A. \& Wiegers, G. A. Chalcogenide misfit layer compounds. J. Alloy. Compd. 229, 144-157 (1995).

12. Cong, Y. et al. Boosting the thermoelectric performance of misfit layered $(\mathrm{SnS})_{1.2}\left(\mathrm{TiS}_{2}\right)_{2}$ by a Co- and Cu-substituted alloying effect. J. Mater. Chem. A 6, 22909-22914 (2018).

13. Kacmarcik, J. et al. Intrinsic Josephson junction behaviour of the low $\mathrm{T}_{\mathrm{c}}$ superconductor $(\mathrm{LaSe})_{1.14}\left(\mathrm{NbSe}_{2}\right)$. Phys. C 468, 543-546 (2008)

14. Cho, N. et al. Crystal structural, electric and magnetic studies on the misfit layer compounds "LnMS 3 " ( $\mathrm{Ln}=$ rare-earth metal; $\mathrm{M}=\mathrm{Ti}, \mathrm{V}, \mathrm{Cr})$. Solid State Ion 63-65, 696-701 (1993).

15. Grosse, C. et al. Selenide/niobium diselenide ferecrystals. Cryst. Res. Technol. 52, 1700126 (2017).

16. Roesky, R., Meerschaut, A., Gressier, P. \& Rouxel, J. Structure parameters, magnetic and electronic properties of bilayer misfit compounds; metalniobium sulfides ["LnNb $\mathrm{S}_{5}$ "; $\mathrm{Ln}=\mathrm{La}, \mathrm{Ce}, \mathrm{Nd}, \mathrm{Sm}, \mathrm{Gd}, \mathrm{Er}$ and Also Y]. Mater. Res. Bull. 29, 943-952 (1994).

17. Hernan, L. et al. X-ray diffraction, XPS, and magnetic properties of lanthanide-based misfit-layered sulfides intercalated with cobaltocene. Chem. Mater. 12, 3792-3797 (2000).

18. Hamann, D. M., Hadland, E. C. \& Johnson, D. C. Heterostructures containing dichalcogenides-new materials with predictable nanoarchitectures and novel emergent properties. Semicond. Sci. Technol. 32, 093004 (2017).

19. Weigers, G. A., Meetsma, A., Haange, R. J. \& Boer, J. L. Structure, electrical transport and magnetic properties of the misfit layer compound $(\mathrm{SmS})_{1.19} \mathrm{TaS}_{2}$ "SmTaS,". J. Less-Common Met. 168, 347-359 (1991).

20. Nader, A., Lafond, A., Briggs, A., Meerschaut, A. \& Roesky, R. Structural characterization and superconductivity in the misfit layer compound (LaSe) ${ }_{1.14}\left(\mathrm{NbSe}_{2}\right)$. Synth. Met. 97, 147-150 (1998).

21. Radovsky, G. et al. Tubular structures from the $\mathrm{LnS}_{-\mathrm{TaS}}(\mathrm{Ln}=\mathrm{La}, \mathrm{Ce}, \mathrm{Nd}$, Ho, Er) and LaSe-TaSe 2 misfit layered compounds. J. Mat. Chem. C 4, 89-98 (2016).

22. Moelo, Y., Meerschaut, A., Rouxel, J. \& Auriel, C. Precise analytical characterization of incommensurate sandwiched layered compounds $[(\mathrm{Pb}, \mathrm{Sn})$ $\mathrm{S}]_{1+x}\left[(\mathrm{Nb}, \mathrm{Ti}) \mathrm{S}_{2}\right]_{m}(0.08 \leq 5 \leq 0.28, m=1-3)$. Role of cationic coupling on the properties and the structural modulation. Chem. Mater. 7, 1759-1771 (1995).

23. Balema, V. P., Wiench, J. W., Pruski, M. \& Pecharsky, V. K. Solvent-free mechanochemical synthesis of two Pt complexes: cis- $\left(\mathrm{Ph}_{3} \mathrm{P}\right)_{2} \mathrm{PtCl}_{2}$ and cis$\left(\mathrm{Ph}_{3} \mathrm{P}\right)_{2} \mathrm{PtCO}_{3}$. Chem. Commun. 15, 1606-1607 (2002). 
24. Singh, N. K., Hardi, M. \& Balema, V. P. Mechanochemical synthesis of an Yttrium based metal-organic framework. Chem. Commun. 49, 972-974 (2013).

25. Auriel, C., Roesky, R., Meerschaut, A. \& Rouxel, J. Structure determination and electrical properties of a new misfit layered selenide $\left[(\mathrm{PbSe})_{1.10} \mathrm{NbSe}_{2}\right]$. Mater. Res. Bull. 28, 247-254 (1993).

26. Suzuki, K. \& Enoki, T. Synthesis, characterization and physical properties of incommensurate layered compounds/(RES) ${ }_{x} \mathrm{TaS}_{2}(\mathrm{RE}=$ Rare Earth Metal). Solid State Commun. 78, 73-77 (1991).

27. Harada, K. \& Ohtani, T. Preparation and properties of novel intergrowth system of BiS $\left(\mathrm{VS}_{2}\right)_{n}(1 \leq n \leq 15)$. J. Jpn. Soc. Powder Metall. 55, 185-191 (2008).

28. Demko, B. A. \& Wasylishen, R. E. Solid-state Selenium-77 NMR. Prog. Nucl. Magn. Reson. Spectrosc. 54, 208-238 (2009).

29. Kemp, T. F., Wong, A., Smith, M. E., Bishop, P. T. \& Carthey, N. A natural abundance ${ }^{77}$ Se solid-state NMR study of inorganic compounds. Solid State Nucl. Magn. Reson. 34, 224-227 (2008).

30. Borsa, F., Torgeson, D. R. \& Shanks, H. R. Charge-density wave amplitudes in $2 \mathrm{H}-\mathrm{NbSe}_{2}$ and $2 \mathrm{H}-\mathrm{TaSe}_{2}$ determined by ${ }^{77} \mathrm{Se}$ NMR. Phys. Rev. B 15, 4576-4579 (1977).

31. Kisoda, K. et al. Raman scattering from misfit layer compounds $(\mathrm{RS})_{x} \mathrm{TaS}_{2}(\mathrm{R}$ identical to La, Ce, Sm or Gd; S identical to Sulphur; $x$ approximately $=1.2$ ). J. Phys. Condens. Matter 7, 5383-5393 (1995).

32. Serra, M. et al. Synthesis and characterization of nanotubes from misfit $(\mathrm{LnS})_{1}$ ${ }_{+y} \mathrm{TaS}_{2}(\mathrm{Ln}=\mathrm{Pr}, \mathrm{Sm}, \mathrm{Gd}, \mathrm{Yb})$ compounds. Chemistry 24, 11354-11363 (2018).

33. Chowalla, M. et al. The Chemistry of two-dimensional layered transition metal dichalcogenide nanosheets. Nat. Chem. 5, 263-275 (2013).

34. Henkelman, G., Arnaldsson, A. \& Jónsson, H. A Fast and robust algorithm for Bader decomposition of charge density. Comput. Mater. Sci. 36, 354-360 (2006).

35. Ziegler, W. T., Brucksch, W. F. \& Hickman, J. W. Studies in superconductivity III. Sn, Cb, Ta, and Pb wires. Phys. Rev. 62, 354-356 (1942).

36. Weber, R. T. The effect of annealing and gas content on the superconducting properties of tantalum. Phys. Rev. 72, 1241-1245 (1947).

37. Revelli, J. F. \& Phillips, W. A. Studies of the system $\mathrm{TaS}_{2-x} \mathrm{Se}_{x}$. J. Solid State Chem. 9, 176-186 (1974).

38. Roisnel, T. \& Rodriguez-Carvajal, J. WinPLOTR: a Windows tool for powder diffraction pattern analysis. Mater. Sci. Forum 378-381, 118-123 (2001).

39. Bhattacharyya, R. \& Frydman, L. Quadrupolar nuclear magnetic resonance spectroscopy in solids using frequency-swept echoing pulses. J. Chem. Phys. 127, 194503 (2007).

40. O’Dell, L. A. \& Schurko, R. W. QCPMG using adiabatic pulses for faster acquisition of ultra-wide line NMR spectra. Chem. Phys. Lett. 464, 97-102 (2008).

41. O'Dell, L. A. The WURST kind of pulses in solid-state NMR. Solid State Nucl. Magn. Reson. 55, 28-41 (2013).

42. Kresse, G. \& Hafner, J. Ab Initio molecular dynamics for liquid metals. Phys. Rev. B 47, 558-561 (1993).

43. Kresse, G. \& Joubert, D. From Ultrasoft pseudopotentials to the projector augmented-wave method. Phys. Rev. B 59, 1758-1775 (1999).

44. Blöchl, P. E. Projector augmented-wave method. Phys. Rev. B 50, 17953-17979 (1994).

45. Monkhorst, H. J. \& Pack, J. D. Special points for Brillouin-zone integrations. Phys. Rev. B 13, 5188-5192 (1976).

46. Perdew, J. P., Burke, K. \& Ernzerhof, M. Generalized gradient approximation made simple. Phys. Rev. Lett. 77, 3865-3868 (1996).

47. Jain, A. et al. Commentary: the materials project: a materials genome approach to accelerating materials innovation. APL Mater. 1, 011002 (2013).

\section{Acknowledgements}

The experimental synthesis work was performed under the auspices of the 2019 laboratory-directed research and development (LDRD) program at Ames Laboratory. Theory support, solid-state NMR spectroscopy, structural and physical property characterization efforts were funded by the US Department of Energy (DOE) Office of Science, Basic Energy Sciences, Materials Science and Engineering Division. Ames Laboratory is operated for the US DOE by Iowa State University of Science and Technology under Contract No. DE-AC02-07CH11358. We thank Dr. Olga Brontvein and Prof. Reshef Tenne from Weizmann Institute, and Dr. Tao Ma from the University of Michigan for assistance and great discussions.

\section{Author contributions}

O.D., I.Z.H., and E.M.E. contributed to the design of experiments, performed material synthesis, material characterization, and contributed to interpretation of results; O.D. and I.Z.H. contributed to writing the manuscript; O.D. performed structural refinements B.W.B., J.L., and E.A.S. performed Raman spectroscopy characterization; A.K.P. and Y M. performed electronic transport experiments and data interpretation; V.K.P. contributed to the structural data evaluation and to writing the manuscript; P.S. and D.D.J. performed DFT calculations and contributed to writing the manuscript; S.L.C. and A.J.R performed solid-state NMR experiments, L.Z. carried out STEM imaging and EDS mapping; V.P.B. conceived the idea, supervised and guided the study, contributed to design of experiments, data interpretation, and the manuscript writing. All authors provided comments and edits during the preparation of the manuscript.

\section{Competing interests}

The authors declare no competing interests.

\section{Additional information}

Supplementary information is available for this paper at https://doi.org/10.1038/s41467020-16672-0.

Correspondence and requests for materials should be addressed to V.P.B

Peer review information Nature Communications thanks anonymous reviewers for their contributions to the peer review of this works. Peer review reports are available.

Reprints and permission information is available at http://www.nature.com/reprints

Publisher's note Springer Nature remains neutral with regard to jurisdictional claims in published maps and institutional affiliations.

(c) (i) Open Access This article is licensed under a Creative Commons By Attribution 4.0 International License, which permits use, sharing, adaptation, distribution and reproduction in any medium or format, as long as you give appropriate credit to the original author(s) and the source, provide a link to the Creative Commons license, and indicate if changes were made. The images or other third party material in this article are included in the article's Creative Commons license, unless indicated otherwise in a credit line to the material. If material is not included in the article's Creative Commons license and your intended use is not permitted by statutory regulation or exceeds the permitted use, you will need to obtain permission directly from the copyright holder. To view a copy of this license, visit http://creativecommons.org/ licenses/by/4.0/.

This is a U.S. government work and not under copyright protection in the U.S.; foreign copyright protection may apply 2020 